\title{
Resignificando el saber pedagógico: una mirada desde la práctica docente
}

\section{Resumen}

\author{
Eugenio Merellano-Navarro ${ }^{1}$ \\ https://orcid.org/0000-0002-1557-2285 \\ Alejandro Almonacid-Fierro² \\ https://orcid.org/0000-0002-8328-017X \\ $\mathrm{M}^{\mathrm{a}}$ Francisca Muñoz Oyarce ${ }^{1}$ \\ https://orcid.org/0000-0003-2027-2866
}

El presente artículo tiene como propósito comprender e interpretar la relación entre la práctica pedagógica desplegada y el saber pedagógico de profesores nóveles ${ }^{3}$ durante el periodo de Formación Inicial Docente (FID), saberes que luego son construidos y desplegados en el quehacer profesional de aula. Para recabar la información se realizaron seis grupos focales y once entrevistas en profundidad a profesores egresados de las carreras de la Facultad de Educación de la Universidad Autónoma de Chile, sede Talca. A partir del análisis de contenido en el marco de la Teoría Fundamentada, se obtuvo como resultado algunas categorías relevantes como las siguientes: significado de los procesos reflexivos durante la formación universitaria, rol docente que favorece el desarrollo de competencias lúdicas, y una alta valoración de las prácticas tempranas durante el proceso formativo. Se concluye que la reflexión permite repensar el despliegue profesional, y consecuentemente la construcción del saber pedagógico. Por su parte, las prácticas tempranas se constituyen en una instancia que permite re-mirar los procesos educativos, a objeto de diseñar estrategias de mejora.

\section{Palabras clave}

Formación de profesores - Saber pedagógico - Aprendizaje - Práctica pedagógica.

\footnotetext{
1- Universidad Autónoma de Chile, Talca, Chile. Contactos: emerellanon@uautonoma.cl; maria.munoz@uautonoma.cl.

2- Universidad Católica del Maule, Chile. . Contacto: aalmonacid@ucm.cl.

3- Profesores nóveles son los que poseen menos de 5 años de experiencia profesional en el contexto escolar.
}

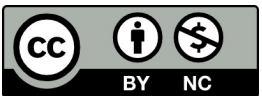




\section{Renaming the pedagogical knowledge: a look from the teaching practice}

\section{Abstract}

The purpose of this article is to understand and interpret the relationship between the pedagogical practice deployed and the pedagogical knowledge of novice teachers ${ }^{4}$ during the period of Basic Teacher Training (BTT), knowledge that is then constructed and deployed in the classroom. To gather information, six focus groups were conducted as well as 11 in-depth interviews with teachers who graduated from the School of Education of the Universidad Autónoma de Chile, Talca. The outcome of data analysis based on Grounded Theory was the emergence of some relevant categories such as: meaning of reflective processes during the undergraduate education; teaching role that benefits the development of recreational skills, and a high appreciation of early practices during the formative process. Conclusion was that the reflection allows to rethink the professional deployment, and consequently the construction of the pedagogical knowledge. In addition, the early teaching practices become a stage that allows novice teachers to re-evaluate the educational processes, in order to design strategies for improvement.

\section{Keywords}

Teacher training - Pedagogical knowledge - Learning - Pedagogical practice.

\section{Introducción}

La Educación está viviendo un proceso de transformación histórica como resultado de las necesidades sociales y la reformulación de los sistemas establecidos orientados a responder a criterios de calidad, acordes con el nuevo escenario económico-social que viven los países (ÁVALOS, 2014). El profesor, desde este escenario, debe asumir nuevos roles en la educación, necesitando en su etapa formativa procesos acordes a la sociedad en la que se vive. En este contexto, conocer los saberes que definen la profesión docente es clave para una efectiva formación de profesores (CÁRDENAS et al., 2012).

El saber pedagógico se define como un conjunto de conocimientos que utilizan los profesores en su despliegue en el aula, instalándose en el modo en como el profesor realiza su trabajo (FRANCIS; MARIN, 2010). La construcción de dicho saber es un proceso individual y colectivo que se inicia en la formación inicial docente, no obstante, se extiende a lo largo de la carrera y ejercicio profesional del profesor. Esta construcción es una responsabilidad compartida entre quienes conforman las comunidades educativas.

4- Novice teachers are those who have less than five years of professional experience in the school system. 
Para ello se requiere un nuevo planteamiento relacional entre teoría y práctica, que incluye reconocer a la práctica como objeto de estudio en permanente elaboración, configurando los espacios educativos y de aprendizaje que generan saber profesional, en la medida que movilizan los saberes de los estudiantes y desarrollan una actitud reflexiva y crítica sobre la base de procesos de análisis disciplinarios, didácticos, metodológicos, pedagógicos y metacognitivos. Al respecto, autores como Graham, Greg y Hastings (2013) y Etscheidt, Curran y Sawyer (2012), describen la enseñanza como una comunidad de práctica emergente, en la que los procesos de reflexión y el diálogo transformador crítico son fundamentales para la mejora de la práctica profesional, proponiendo a su vez que la formación del profesorado debe profundizar en los procesos reflexivos, configurando una propuesta que considere un sólido conocimiento del contenido, instancias sistemáticas de práctica en aula y el desarrollo del pensamiento reflexivo. En esta línea, Moore-Russo y Wilsey (2014) y Mena y García (2013), establecen importantes precisiones a la hora de otorgar significado al rol de la reflexión crítica y las consecuentes representaciones acerca de la enseñanza de profesores en formación y novatos, concluyendo que los estudio referidos a la reflexión crítica por parte del maestro aportan una contribución importante al corpus de investigación sobre la reflexión del maestro al poner de relieve las diferentes dimensiones de la reflexión. En consecuencia, las comunidades de formación docente deberían profundizar seriamente en el significado de la reflexión productiva al considerar el contenido, la conexión y la complejidad de las reflexiones y, por otro lado, apostar por un profesor crítico que sea capaz de interpretar la práctica de modo genuino, a partir de su propia experiencia de aula.

Los planteamientos más recientes en relación a la tarea del profesor en el aula y el sistema escolar dicen relación en comprender al profesor como un profesional reflexivo, lo que implica que el docente en lugar de limitarse a replicar mecánicamente soluciones ensayadas por otros para resolver las situaciones que se plantean en la práctica pedagógica, indaga constantemente soluciones creativas y pertinentes a la realidad de sus estudiantes, al contexto particular, en una acción que implica activar tanto los conocimientos teóricos adquiridos en la formación inicial docente (FID), como aquellos que surgen de su experiencia individual y colectiva de enseñanza. En este sentido, podemos señalar que la reflexión en la enseñanza, particularmente desde los aportes de Donald Schön (1998), es un tema relevante en la literatura relacionada con los docentes, toda vez que la reflexión ha sido aceptada como parte integral de los procesos de formación del profesorado, tanto desde el punto de vista teórico, como desde una perspectiva práctica de la acción en el aula (BEAUCHAMP, 2015; SCHÖN, 2010).

Por otro lado, el profesor es considerado actualmente un profesional que revisa y cuestiona críticamente su hacer didáctico, y las implicaciones educativas que subyacen a su práctica de aula, a través de un ejercicio de reflexión en la acción y reflexión sobre la acción. Al respecto, Ruffinelli (2017) presenta una exhaustiva revisión bibliográfica acerca del enfoque del profesor como profesional reflexivo y como la idea de reflexividad se instala en la investigación pedagógica de los últimos 25 años, constituyéndose en uno de los ejes centrales del perfil de un profesor que las instituciones formadoras de profesores declaran construir. No obstante, los hallazgos investigativos dan cuenta que los niveles 
de reflexividad en el profesorado en formación se sitúan en niveles más bien descriptivos, con incipientes resultados que se acercan a un profesor crítico y transformador, cuestión que se complementa con los estudios de Mauri, Clará y Onrubia (2016), Castellanos y Yaya (2013) y Garrido (2016).

Todo profesor necesita de interacciones sociales en un contexto determinado para su desarrollo profesional, es por ello que cuando se habla de saber pedagógico, no podemos dejar de lado la influencia de la sociabilización en el docente y como estas interacciones van formando un modo de comprender el mundo por parte del profesor (TARDIF, 2004). Por otro lado, el saber pedagógico no sólo comprende el estudio de los currículos, el conocimiento de la práctica o la propia formación inicial, muy por el contrario, el hilaje del saber pedagógico engloba el mundo del docente en la tarea laboral, la auto observación y seguidamente la reflexión crítica del proceso en la práctica de aula. Por lo tanto, el profesor es un profesional poseedor de un saber que proviene de su formación inicial y preferentemente de su acción docente. Asume su práctica pedagógica a partir de los significados que él mismo le otorga, cuestión que conduce a orientar y/o reorientar su acción pedagógica (LATORRE, 2002; FLORES, 2005; TARDIF, 2004; BARRON, 2006). En este sentido, el quehacer docente está caracterizado por una serie de aprendizajes que permiten a los profesores comprender y explicar la realidad profesional a partir de sus propias acciones, considerando dos elementos centrales: las perspectivas epistemológicas y el accionar didáctico, pues estos dos elementos facilitan la orientación de dichos saberes profesionales (FREIRE; FERNÁNDEZ, 2015).

En este contexto, el saber pedagógico implica el ejercicio de una práctica reflexiva, comprometida, con sentido e intencionalidad. Requieren la presencia de un sujeto capaz de generar una relación entre teoría y práctica, puesto que la acción docente se configura en torno a una praxis en diálogo permanente entre ambos dominios, generando una amalgama de conocimientos que emergen desde la práctica hacia la teoría y desde la teoria hacia la práctica. En consecuencia, es a partir de este ejercicio sistemático y recursivo que surge un conocimiento de carácter curricular, didáctico, disciplinar y reflexivo, denominado en términos genéricos saber pedagógico que, en definitiva, permite generar conocimiento desde la propia práctica pedagógica, a objeto de poder transformar esa misma práctica (SCHÖN, 1998, 2010; FREIRE, 2002; CORNEJO; FUENTEALBA, 2008; CARDONA, 2011; FRAILE, 2011; MONTENEGRO; FUENTEALBA, 2010; ERAZ0, 2009; MARCELO, 2009). El profesor se transforma en generador de conocimiento al plasmar la instancia reflexiva desde la propia práctica, reconstruyéndola y resignificándola. Se entiende, entonces, que es en el aula escolar el lugar donde se planifica, organiza, ejecuta y evalúa el proceso educativo y, por tanto, es ahí donde se configura, deconfigura y finalmente reconfigura el saber pedagógico, entendido como “[...] los conocimientos, construidos de manera formal e informal por los docentes; valores, ideologías, actitudes, prácticas; es decir, creaciones del docente, en un contexto histórico cultural" (DIAZ, 2006, p. 95).

En este sentido, la presente investigación se justifica en términos de revisar y analizar los aprendizajes de los procesos de formación inicial desde un punto de vista crítico, con la idea de comprender de qué manera los profesores noveles se apropian del saber pedagógico, particularmente a través de su incorporación al sistema escolar, y al 
ejercicio docente de aula, lugar y momento, en que construye y reconstruye sus saberes docentes (ÁVALOS; CARLSON; AYLWIN, 2005). La novedad de este trabajo se debe a que reúne profesionales noveles de diferentes disciplinas educativas que están insertos en el ámbito escolar, entregando una visión global y multidisciplinar del contexto escolar. Por otro lado, el estudio aborda la categoría saber pedagógico y desde esta dimensión se analiza la reflexión sobre la práctica, desde la perspectiva de docentes que se incorporan al sistema educativo. En términos metodológicos, si bien es cierto la mayoría de los estudios en esta temática tiene un carácter cualitativo, en la presente investigación se aplican dos técnicas que se complementan y permiten enriquecer la comprensión del fenómeno.

El presente artículo es parte de una investigación mayor que busca comprender e interpretar las relaciones entre la práctica pedagógica desplegada y el saber pedagógico de profesores nóveles. Específicamente, se expondrá la segunda categoría que emerge de la investigación, denominada Aprendizaje en la formación inicial, categoría que nos permite comprender cuáles son los aprendizajes construidos en la formación docente, específicamente, los saberes pedagógicos aprendidos en la etapa universitaria, entendidos éstos como los conocimientos construidos formal e informalmente, desarrollados en los procesos de formación inicial, y que luego se despliegan en el contexto laboral. Estos saberes están compuestos por valores, ideologías, actitudes y prácticas que el profesorado despliega en su actuación pedagógica en el aula, en el ejercicio específico de la docencia (ALMONACID; MERELLANO; MORENO, 2014; SANTANA; DALMAZO, 2012; ALLIAUD; VEZUB, 2012; ERAZ0, 2009).

\section{Metodología}

El presente estudio adopta como opción la metodología cualitativa, toda vez que la investigación pretende la comprensión subjetiva de los sujetos en relación a los saberes pedagógicos adquiridos en su proceso formativo y que luego son transmitidos en su despliegue laboral (RODRÍGUEZ; GIL; GARCIA, 1999; VASILACHIS, 2006; GIBBS, 2012).

Se utilizó en esta investigación el estudio de caso, el cual tiene como propósito “[...] investigar la particularidad, la unidad, del caso singular” (SIMONS, 2011, p. 20). En este sentido el estudio se desarrolló en la Facultad de Educación de la Universidad Autónoma de Chile, sede Talca, en la que se imparten las carreras de Pedagogía en las siguientes especialidades: Artes Visuales, Matemática, Inglés, Lengua Castellana y Comunicación, Educación Física, Educación General Básica, Historia Geografía y Ciencias Sociales y Educación Parvularia.

Se procedió a realizar once entrevistas en profundidad y seis grupos focales donde participaron las ocho carreras que componen la Facultad de Educación (ver tabla 1).

Para el reclutamiento de las personas que participaron en el estudio se solicitó a cada carrera de la Facultad de Educación que facilitara información de sus egresados, con el fin de contactarlos telefónicamente e invitarlos a participar en el estudio. La selección de la muestra fue por conveniencia, intentado fomentar heterogeneidad en relación a número, género, dependencia escolar, año de egreso y ciudad. Todos los participantes fueron citados un día de la semana laboral en horario 18-20 horas, en dependencias de 
la Universidad. Las citaciones se organizaron en función de carrera. Solo seis de las ocho carreras obtuvieron el número mínimo para la ejecución del grupo focal (seis participantes).

Posteriormente a la realización de cada grupo focal, se seleccionaron informantes clave de cada una de las carreras que cumplieron con el mínimo de participantes. Los informantes clave fueron citados una semana después en la sala de reuniones de la facultad para realizarles individualmente una entrevista en profundidad. Las dos carreras que no lograron el mínimo de participantes en los grupos focales se optaron por entrevistar a todos los que llegaron a la primera citación.

Todos los sujetos que participaron en el estudio tuvieron que cumplir los siguientes criterios:

- Profesional egresado de la universidad durante el periodo 2008-2013.

- Accesibilidad: Desempeñarse profesionalmente en establecimientos educacionales de la región del Maule-Chile.

- Interés en participar en el estudio.

Tabla 1- Distribución por carrera e instrumentos de recolección de datos

\begin{tabular}{|c|c|c|c|c|}
\hline CARRERAS & FOCUS GROUP & $\begin{array}{l}\text { PARTICIPANTES } \\
\text { FOCUS GROUP }\end{array}$ & ENTREVISTAS & OBSERVACIONES \\
\hline Pedagogía en Artes & 1 & 6 & 1 & \\
\hline Pedagogía en Educación Básica & - & - & 2 & Sin Focus Group \\
\hline Pedagogía en Matemáticas & 1 & 7 & 1 & \\
\hline Pedagogía en Educación Física & 1 & 6 & 2 & \\
\hline Pedagogía en Historia / Geografía & 1 & 8 & 1 & \\
\hline Pedagogía en Inglés & - & - & 2 & Sin Focus Group \\
\hline Pedagogía en Castellano & 1 & 6 & 1 & \\
\hline Educ. Parvularia & 1 & 8 & 1 & \\
\hline Total & 6 & 41 & 11 & \\
\hline
\end{tabular}

Fuente: Elaboración propia.

\section{Proceso de recolección y análisis de la información}

Luego de la realización de las entrevistas y grupos focales se llevó a cabo la transcripción de dichos instrumentos, categorización de la información, sistematización, análisis y reducción de los datos, siguiendo las recomendaciones de Flores (2009), Kvale (2011) y Taylor y Bogdan (1984).

El proceso de reducción de la información se realizó utilizando la técnica de análisis de contenido por medio de la codificación de los datos obtenidos, para luego construir la matriz de sistematización. El proceso de categorización comenzó con la revisión del marco teórico, ya que es desde estos referentes que se levantaron las categorías previas. Posteriormente, se inició el proceso de codificación, tal como lo presentan Coffey y Atkinson (2003, p. 32) “[...] los códigos vinculan diferentes segmentos o ejemplos presentes en los 
datos. Traemos estos fragmentos de los datos y los reunimos para crear categorías que definimos con base en alguna propiedad". Posteriormente, se ramificaron las categorías previas por medio de una codificación selectiva (STRAUSS; CORBIN, 2002), permitiendo el nacimiento de las categorías primarias. Todo lo anterior, permite la comprensión que los profesores noveles otorgan a la dimensión saber pedagógico en su proceso de formación inicial, y seguidamente en el espacio escolar de aula.

Los datos investigados se organizan en la siguiente matriz (ver tabla 2). En este trabajo, se expondrán los resultados más significativos de la segunda Categoría Previa Aprendizajes en la formación inicial.

\section{Resultados}

Tabla 2- Matriz sistematización ${ }^{5}$

\begin{tabular}{|c|c|c|c|}
\hline MEGACATEGORÍA & CATEGORÍAS PREVIAS & DESCRIPTOR & CATEGORÍA PRIMARIA \\
\hline \multirow{3}{*}{$\begin{array}{c}\text { Saberes desplegados por los } \\
\text { egresados de la facultad de } \\
\text { educación Universidad Autónoma } \\
\text { de Chile }\end{array}$} & Tipos de Saberes & $\begin{array}{l}\text { Categoría que alude a } \\
\text { los tres dominios de este } \\
\text { saber; reflexivo, laboral y } \\
\text { conceptual. }\end{array}$ & $\begin{array}{c}\text { Saber Reflexivo } \\
\text { Saber Curricular o Conceptual }\end{array}$ \\
\hline & $\begin{array}{l}\text { Aprendizajes en la } \\
\text { formación inicial }\end{array}$ & $\begin{array}{l}\text { Saberes pedagógicos, } \\
\text { emergencia en el transcurso } \\
\text { de la línea de formación } \\
\text { práctica }\end{array}$ & $\begin{array}{c}\text { Reflexión en la Práctica } \\
\text { Aspectos y-o Ámbitos Disciplinares } \\
\text { Métodos de Enseñanza y Aprendizaje } \\
\text { Ejercicio Profesional } \\
\text { Ethos Docente } \\
\text { Diversidad en el Área Educativa }\end{array}$ \\
\hline & $\begin{array}{l}\text { Sugerencias para } \\
\text { la formación del } \\
\text { profesorado }\end{array}$ & $\begin{array}{l}\text { Propuestas para la mejora de } \\
\text { la formación docente }\end{array}$ & $\begin{array}{l}\text { Enfocadas en el Currículo } \\
\text { Enmarcadas en el Área de Formación Práctica } \\
\text { Relacionada con el Proceso de Formación } \\
\text { Relacionada con el Profesorado } \\
\text { Relacionada con la Comunicación } \\
\text { Relacionada con la Evaluación }\end{array}$ \\
\hline
\end{tabular}

Fuente: Elaboración propia a partir del análisis de los datos.

Los datos de las fuentes primarias, vale decir focus group y entrevistas, se analizaron de manera inductiva con constantes análisis y comparaciones, lo que permite organizar y categorizar los datos a través del análisis de contenido. En este sentido, el análisis lo hemos estructurado bajo una lógica en que en un primer momento define la categoría primaria, se exponen algunos relatos provenientes de los documentos primarios y seguidamente se conecta con algunos referentes teóricos a través de una reflexión crítica.

\footnotetext{
5- Matriz con la totalidad de las categorías de la investigación. En este trabajo se analizó la categoría previa Aprendizajes en la formación inicial. El proceso de análisis y reducción de datos permitió que, a partir de la mega categoría, se encontrasen las categorías más representativas del discurso (categorías primarias), agrupándolas en tres categorías previas. Cada categoría se clasificó en relación a las dimensiones del estudio, cuestión que nos introduce en un proceso dialectico y recursivo de construcción de significados.
} 
A partir de aquí trataremos de dar cuenta de cada una de las categorías que emergen del proceso de análisis y reducción de datos (ver Tabla 2), para finalmente responder al propósito del estudio que dice relación con comprender e interpretar las relaciones entre la práctica desplegada y el saber pedagógico de los docentes investigados.

\section{Categoría previa Aprendizaje en la Formación Inicial}

\section{Categoría primaria 1: reflexión en la práctica}

Los relatos se presentarán con su respectivo código, con la intención de tener un rápido acceso al documento original. Cada uno de los códigos estará entre paréntesis e incluirá la siguiente información: en primer lugar, el tipo de recurso y numero respectivo, seguidamente del subcódigo de la transcripción y finalmente la página donde se encuentra el relato.

Yo recuerdo que en la Universidad se daba mucho énfasis sobre la reflexión. Yo creo que eso me dejo (...) bien marcado (...). (Focus Group 3, f1-p. 1).

En el marco de la categoría previa Aprendizaje en la formación inicial, los sujetos investigados dan cuenta de un proceso de Reflexión en la práctica, al expresar que el currículo de formación profesional en el área de formación pedagógica de la Facultad de Educación, presenta un componente reflexivo importante, cuestión que vemos reflejada en los programas de estudios implementados en las diferentes carreras, específicamente en los procesos de aproximación e inserción escolar, puesto que es en estas instancias donde se facilitan las relaciones con sus pares mediante el diálogo y los espacios de retroalimentación conforme a las experiencias vividas por cada uno de los docentes (MENA; GARCIA, 2013). No obstante, en el relato antes mencionado, no se clarifica el tipo de profundidad de las acciones reflexivas desarrolladas en la formación inicial por la Facultad de Educación, similar a lo descrito por Ruffinelli (2017). Un profesional reflexivo posee un criterio experto y meditado en el ámbito de la docencia, a través de un repensar de su propio despliegue en el aula, por lo tanto, los espacios que la Universidad dispone para desarrollar reflexión en torno a la acción o en la acción pedagógica, deberán potenciar instancias que trasciendan a los momentos individuales e incorporar procesos de acción colectiva y trabajo de equipo en diferentes contextos. Este proceso requiere ser aprendido y ejecutado en la etapa formativa $\mathrm{y}$, de esta forma, generar una actitud y habilidad que potencie la acción intencionada en el ámbito profesional (FULLAN; HARGREAVES, 2006).

Creo que debemos los profesores ser autocríticos (...) podemos mejorar nuestras prácticas siempre y cuando haya reflexión, creo que son muchos los profesores o somos muchos los que nos creemos casi omnipotentes, que no cometemos errores y si no hay reflexión no hay mejoras y si no hay mejoras no hay aprendizaje. (Entrevista 5, f114-p. 224). 
A través del proceso reflexivo, se desarrolla en los estudiantes un pensamiento crítico e investigativo de su propio despliegue en el aula, favoreciendo de esa forma mejores experiencias de aprendizajes en sus estudiantes. Este reflexionar crítico favorece el descubrimiento de nuevas formas para aprender y enseñar. No obstante, los hallazgos investigativos de Beauchamp (2015), dan cuenta que los formadores de docentes no han brindado la claridad adecuada sobre el significado de la reflexión y han mantenido su enfoque fuera del aula, cuestión que impacta en los primeros años de ejercicio docente, al no existir continuidad en el aprendizaje profesional y continuo del profesorado.

Es así como el proceso reflexivo se hace relevante en toda práctica laboral educativa, puesto que éste es el que facilita las relaciones con sus pares, mediante el diálogo y los espacios de retroalimentación, instancias que deberían darse tanto en la FID, como en el desarrollo profesional docente (MONTENEGRO; FERNÁNDEZ, 2015; DOMINGO ROGET; GÓMEZ SERÉS, 2014; ASTUDILLO; RIVAROSA; ORTIZ, 2014). Además, se observa la relevancia de este trabajo mutuo por parte del conocimiento que emerge en las prácticas pedagógicas como a su vez en los procesos reflexivos insertos en cada instancia educativa, provocando momentos de retroalimentación, enriquecimiento personal y colaboración en las instancias de inserción docente.

\section{Categoría primaria: aspectos y-o ámbitos disciplinares}

[...] evaluación, ahora segundo ciclo, psicología del desarrollo y del aprendizaje, (...), lenguaje y comunicación, (...) no recuerdo si uno se llamaba matemática o lógico matemática, que también es súper importante, un ramo que se llamaba ciencias, pero los conocimientos que nos entregaron ahí no eran muy buenos, así que tuve que investigar por las mías no más. (Focus Group 6, F59-p. 114).

En el análisis de la categoría primaria Aspectos y/o ámbitos disciplinares se constata la gama de disciplinas que la universidad entrega a sus estudiantes de Pedagogía, cuestión que se traduce en un itinerario curricular o plan de estudios, que desarrolla en los educandos las competencias pedagógicas y disciplinares que posteriormente aplicarán en el sistema escolar.

En este sentido Ávalos (2002), menciona que la estructura de las mallas de las universidades chilenas en la formación de profesores, de acuerdo a las ofertas curriculares en las áreas tradicionales, se definieron en cuatro dimensiones o áreas: general, especializada, profesional y práctica. Uno de los informantes clave hace mención en su relato que una de las asignaturas o módulos cursados, específicamente el de ciencias, no contribuyo a su formación como estudiante, debiendo recurrir al aprendizaje autónomo a través de la indagación personal, logrando aprendizajes débiles y no aplicables en su despliegue laboral. No obstante, los egresados de la facultad expresan que al no dominar ciertos conocimientos pedagógicos se ven en la necesidad de investigar por sus propios medios con el fin de profundizar lo que no fue entregado por la universidad. Esta capacidad de investigación es propia de un docente comprometido con su acción pedagógica (SANTIAG0; PARRA; MURILLO, 2012). 
[...] motricidad, (...) ¿qué más? todo, todo, el lenguaje, la matemática, yo tengo que saber de todo eso, para que el niño pueda aumentar su conocimiento y obtenga un progreso que esté acorde a su edad, porque si yo no estoy bien capacitada obviamente no voy a entregar un buen aprendizaje, un buen conocimiento y el niño va a disminuir sus posibilidades de desarrollo. (Entrevista 8, f60-p. 115).

Como se menciona, el conocimiento de todas las disciplinas es necesario para que los niños aprendan y aumenten progresivamente sus competencias en la escuela. Es por esto que la Universidad en la elección de su currículo enseña en profundidad una gran variedad de disciplinas para preparar correctamente a los futuros docentes. Se plantea que para desarrollar la identidad docente y el conocimiento y capacidades del profesorado, la institución formadora debe establecer estructuras y estrategias que permitan transformar el saber en conocimientos enseñables, por lo que las disciplinas aprendidas en la universidad son necesarias para que posteriormente puedan ser enseñadas en las aulas de escuelas y liceos del país (ÁVALOS; CARLSON; AYLWIN, 2005).

\section{Categoría primaria: métodos de enseñanza y aprendizaje}

[...] nosotros teníamos didácticas de todas las asignaturas, y esas didácticas no iban directamente referidas a cómo hacer un material, construir materiales, construir monitos, pasarlo bien, sino que íbamos más bien a adoptar un modelo, adoptar distintas metodologías, poder optar también por distintas estrategias. (Focus Group 2, f82-p.141).

En el marco de la categoría de Métodos de enseñanza y aprendizaje, los profesionales egresados mencionan que el ámbito didáctico que recibieron dentro de su formación docente durante su transcurso universitario les fue relevante para proyectar los diversos contenidos, manifestándolo en el relato presentado. El conocimiento didáctico se considera necesario para facilitar el aprendizaje integral de los estudiantes, debido al diseño potencial de medios formativos que éste proporciona, caracterizándose por su gran enfoque innovador entre el fundamento teórico-práctico, cuya implicancia procura preservar una sistematización rigurosa y pertinente frente al quehacer pedagógico. Seguidamente, dentro de esta categoría previa, se menciona la importancia del juego como herramienta facilitadora del aprendizaje, como se aprecia en el siguiente relato:

[...] el juego, yo creo que es la estrategia más valida o más efectiva para los niños pre-escolares o también hasta cuarto básico, yo creo, el juego te abre muchas puertas en cuanto a los niños y al aprendizaje, porque a ellos le encanta jugar, pero, obviamente tienes que llevar bien el juego. (Entrevista 6, f62-p. 118).

Esto deja en evidencia la visión de los sujetos que participaron en el estudio en relación al juego. Esta apreciación se desarrolla por medio de la opción metodológica utilizada por la Facultad de Educación, potenciada por sus currículos y en la cual los contenidos de las distintas asignaturas asociadas a la didáctica disciplinar incorporan 
el valor del juego en la etapa de educación infantil y, principalmente, como agente motivador. No obstante, por la naturaleza de cada disciplina, esta opción lúdica es más aceptada por las carreras de Pedagogía en Educación Física, Pedagogía en Educación de Párvulos y Pedagogía en Educación General Básica. Por otro lado, los sujetos entrevistados manifiestan el uso del juego como una opción que facilita el aprendizaje de los estudiantes. A través de los focus group y entrevistas en profundidad, se puede deducir que el juego proporciona nuevas formas de explorar la realidad y estrategias distintas de conocer, puesto que favorece momentos para la espontaneidad, en un mundo donde la mayoría de las cosas están normadas. No obstante, el rol que debe realizar el docente en el juego es clave a la hora del éxito del mismo. El valor del juego es lograr experiencias positivas en el aprendizaje y no solo por diversión. De acuerdo a lo planteado por Muñoz y Almonacid (2015), el aprendizaje basado en el juego resulta del todo relevante a objeto de que los niños y niñas organicen y den sentido a sus mundos, toda vez que de esta manera se comprometen activamente con las personas, los objetos y las representaciones. En este sentido, el juego con características de físicamente elevado, permite a los niños probar y desarrollar todo tipo de habilidades motoras, generando además múltiples beneficios en el ámbito de la salud.

\section{Categoría primaria: ejercicio profesional}

En el último semestre cuando fue mi práctica (...) hice todas las clases, entonces (...) cuando entré a trabajar, ya tenía muchos conocimientos, ya sabía el manejo que había que tener, entonces me sirvió demasiado lo que es la práctica en sí, mucho más que la teoría. (Focus Group 3, f96-p. 173).

La universidad inserta desde muy temprano a los estudiantes en el ámbito escolar, teniendo éstos desde los primeros años diferentes tipos de aproximaciones e inserciones al contexto escolar. En este sentido la propuesta formativa intenta conectar adecuadamente los dominios teoría y práctica, toda vez que ambas dimensiones van unidas en la experiencia, puesto que uno se construye en base del otro, el conocimiento se adquiere tanto en la práctica laboral como en el contexto universitario. Por ende, tanto la teoría como la práctica, se ligan y religan de manera recursiva. Lo anterior, de acuerdo a lo expuesto por Mauri, Clará y Onrubia (2016), se debe dar en una relación sinérgica entre el tutor y el estudiante en formación, toda vez que esta perspectiva colaborativa y de apoyo, permitiría que los maestros tutores ayuden a los estudiantes a la identificación y análisis de los dilemas que surgen desde la práctica.

Cabe destacar la importancia que le atribuyen los egresados a los procesos de aproximación y práctica, siendo éstas más valoradas que el proceso de formación que reciben en la universidad, principalmente, por los cursos que denominan teóricos. Es importante lograr en los estudiantes un equilibrio en la valoración, construyendo significados desde el aula para luego ser aplicados exitosamente en el ámbito escolar.

Yo puedo articular casi a la perfección los componentes de la planificación, yo considero que planifico muy bien y muy rápido y me cuesta muy poco, y eso fue gracias a la exigencia y 
al transcurso que pase en mi carrera que me fueron pidiendo (...) yo no me enfrente con la planificación el último año, cuando yo llegue a la práctica, me dicen... tienes que planificar, éste es el modelo, ¿qué hago?, no pues yo me enfrente con la planificación desde primero, entonces ahí, uno va adquiriendo más saberes, va adquiriendo más aprendizajes, y los va incorporando a diario. (Entrevista 4, f80-p. 140).

El relato del entrevistado da a conocer que el paso por la universidad le ayudó bastante en el fortalecimiento de las planificaciones de sus clases. La planificación es un instrumento clave de todo proceso docente, se enmarca dentro del orden, organización y conformación de las clases en las escuelas en general. Dentro de esto no se puede dejar de lado que para adquirir un ejercicio profesional se requiere desarrollar competencias desde el inicio del proceso formativo, competencias que se van incorporando al profesor en formación mediante un proceso de enseñanza-aprendizaje (SANTORO, 2008).

\title{
Categoría primaria: ethos docente
}

\begin{abstract}
No sé si ahora estarán en el primero, pero nosotros empezamos ya del segundo año tuvimos aproximación I y II, después inserción I y II, práctica I y práctica profesional, pero es bueno que ese tipo de medidas porque así los alumnos realmente saben si son como acordes a la carrera y si realmente tienen afinidad porque ser profesor como dicen por ahí, es una vocación y ahí uno realmente tiene la capacidad y tiene las aptitudes para poder estar ahí. (Entrevista 11, f117-p. 228).
\end{abstract}

Dentro de la categoría primaria de Ethos docente, surge la necesidad de hacer hincapié en la importancia que tiene el área de formación práctica, instancia formativa en la que se encuentran inmersas las diferentes aproximaciones, hasta finalizar con la práctica profesional.

En el relato presentado, se constata que los egresados valoran la práctica temprana como uno de sus principales elementos de la formación. Asimismo, en relación a la práctica docente, se comparte una concepción más bien gradual de la inserción directa al aula, ya que las primeras intervenciones son de observación, para luego insertarse progresivamente dentro del aula educativo, en donde se vislumbra una fase experimental, cuya orientación permite dar cuenta si el estudiante en formación tiene vocación o no, reconociéndose su identidad profesional como tal, bajo un contexto real de la educación. En este contexto, es de suma importancia que los profesores en formación aprendan a desempeñarse de forma competente e integral frente a las diversas realidades educativas, pero por sobre todo con sentido pedagógico y compromiso frente al acto de enseñar, ya que el profesor asume un rol trascendental en la formación de cientos de niños a la hora de desplegar su intención pedagógica. En este sentido se comprende que el ser humano es un reflejo de toda su vivencia e interacción con el medio ambiente que lo rodea, $\mathrm{y}$ con las interrelaciones sociales que va teniendo a lo largo de su vida, por lo tanto, la identidad profesional de un egresado es el reflejo de sus experiencias de las vivencias en el mundo escolar, tal y como lo plantea Maturana (2011). 


\title{
Categoría Primaria: diversidad en el área educativa
}

\begin{abstract}
A las necesidades educativas especiales, si yo tuve ese ramo, obviamente esa asignatura es importante, ese ramo lo tuve y me costó arto, pero es súper importante porque siempre en los cursos ves que los niños tienen problema del lenguaje, o trastornos, o déficit atencional, entre otras cosas, entonces también tienes que saber tratarlos, o saber a dónde recurrir para que el niño progrese, entonces, es súper importante que sea considerado en la formación. (Focus Group 5, f61-p. 116).
\end{abstract}

La inclusión educativa es hoy una aspiración de todos los sistemas educativos de Iberoamérica y no podría ser de otra manera si aspiramos a que la educación al desarrollo de sociedades más justas, democráticas y solidarias, pero para lograr esto se necesita según Infante (2010, p. 290) "[...] un conjunto de herramientas educacionales que responden a la educación de estudiantes con necesidades educativas especiales derivadas de discapacidad o con trastornos específicos del lenguaje en un contexto de educación regular”. Es por ello el hincapié de colocar dentro del plan de estudios de las carreras de la Facultad de Educación el trabajo con las necesidades educativas especiales, a objeto de entregar las herramientas acordes al desarrollo de una temática absolutamente contingente a nivel de sociedad y sistema educativo.

Consecuentemente, considerando la diversidad según los diferentes contextos, ya sea en el sistema preescolar, básico y medio, de carácter municipal o particular, se estima necesario abordar durante la línea formativa la vivencia en las múltiples realidades que forman parte del sistema escolar actual.

\section{Conclusiones}

La investigación desarrollada enfatiza la reflexión en los procesos de formación inicial en la Facultad de Educación de la Universidad Autónoma de Chile, abordándose metodológicamente como estudio de caso. Es por esto que sus hallazgos investigativos no pretenden ser generalizados.

Se devela desde el discurso de los informantes clave que su ejercicio profesional se encuentra mediado por estrategias de enseñanza-aprendizaje vivenciadas en la FID, $\mathrm{y}$ fundamentalmente por un fuerte componente reflexivo que caracteriza su despliegue pedagógico. Se describe que en el currículo se intencionan momentos de reflexión con el fin de repensar y remirar el despliegue que tienen los profesores en formación en sus procesos de inserción en el aula. A través de este ejercicio se desarrollan capacidades que deben poseer los docentes, tales como la autocrítica y la capacidad investigativa en post de mejorar la práctica educativa.

El estudio enfatiza la relevancia del juego y la innovación dentro del currículo formativo en las asignaturas relacionadas a la didáctica de la especialidad. Se destaca la labor que poseen los profesores en la universidad al fomentar estrategias innovadoras y lúdicas que estimulen a los alumnos a aprender. Esta característica en la formación inicial trasciende en el ejercicio laboral ya que se replican metodologías aprendidas en la FID, 
favoreciendo las experiencias de aprendizaje en el contexto escolar. Específicamente, el juego debe poseer principalmente un carácter pedagógico y no solo lúdico, el que debe estar orientado y guiado en favor del aprendizaje.

La experiencia es reconocida por los egresados como medio de adquisición y corporalización de conocimientos en el contexto escolar y principalmente, el aprendizaje y competencias académicas y pedagógicas necesarias en la formación de un profesor. La experiencia otorgada por la línea de formación práctica permite a los profesionales en formación significar y resignificar los saberes curriculares compartidos en la formación inicial y seguidamente rescatarlos en el contexto escolar. Por lo mismo, se valora la necesidad de instancias de práctica temprana, permitiendo formarse desde los primeros semestres en contacto con el sistema escolar.

Se concluye que los Aprendizajes en la formación inicial son aprendizajes de partida, ya que son la base para afrontar exitosamente el mundo laboral. También poseen la característica de inacabado, al fortalecerse, construirse y reconstruirse por medio de las experiencias, y seguidamente compartidos, ya que el camino de ser profesor se conjuga a partir de la relación con los diferentes actores que favorecen el aprendizaje.

\section{Referencias}

ALLIAUD, Andrea; VEZUB, Lea. El oficio de enseñar. Sobre el quehacer, el saber y el sentir de los docentes argentinos. Revista Diálogo Educacional, Curitiba, v. 12, n. 37, p. 927-952, 2012. Disponible en: <http:// www.redalyc.org/articulo.oa?id=189124308018>. Acceso en: 24 my. 2018.

ALMONACID, Alejandro; MERELLANO, Eugenio; MORENO, Alberto. Caracterización del saber pedagógico: estudio en profesores noveles. Revista Educare, Heredia, v. 18, n. 3, p. 173-190, 2014. Disponible en: <http://www.scielo.sa.cr/pdf/ree/v18n3/a10v18n3.pdf>. Acceso en: 14 jun. 2018.

ASTUDILLO, Carola; RIVAROSA, Alcira; ORTIZ, Félix. Reflexión docente y diseño de secuencias didácticas en un contexto de formación de futuros profesores de ciencias naturales. Perspectiva Educacional, Valparaíso, v. 53, n. 1, p. 130-144, 2014. Disponible en: <http://www.perspectivaeducacional.cl/index. php/peducacional/article/view/128>. Acceso en: 24 my. 2018.

ÁVALOS, Beatrice. La formación inicial docente en Chile: tensiones entre políticas de apoyo y control. Estudios Pedagógicos, Valdivia, v. 40, n. esp. p. 11-28, 2014. Disponible en: <https://scielo.conicyt.cl/ scielo.php?script=sci_arttext\&pid=S0718-07052014000200002>. Acceso en: 24 my. 2018.

ÁVALOS, Beatrice. Profesores para chile, historia de un proyecto. Santiago de Chile: Ministerio de Educación, 2002.

ÁVALOS, Beatrice; CARLSON, Beverley; AYLWIN, María del Pilar. La inserción de profesores neófitos en el sistema educativo: ¿cuánto sienten que saben y cómo perciben su capacidad docente en relación con las tareas de enseñanza asignadas? Santiago de Chile: [s. n.], 2005. Proyecto fondecyt 1020218. Disponible en: <http://www.cepal.org/ddpe/publicaciones/sinsigla/xml/7/19597/INSERPROFE.pdf>. Acceso en: 24 my. 2018. 
BARRÓN, Concepción. Los saberes del docente: una perspectiva desde las humanidades y las ciencias sociales. Revista Perspectiva Educacional, Valparaíso, n. 48, p. 11-26, 2006. Disponible en: <http://www. redalyc.org/pdf/3333/333328829001.pdf>. Acceso en: 24 my. 2018.

BEAUCHAMP, Catherine. Reflection in teacher education: issues emerging from a review of current literature. Reflective Practice, New York, v. 16, n. 1, p. 123-141, 2015. Disponible en: <https://www.tandfonline.com/ doi/abs/10.1080/14623943.2014.982525?journalCode=crep20 >. Acceso en: 19 jul. 2017.

CÁRDENAS, Ana et al. El saber pedagógico: componentes para una reconceptualización. Educación y Educadores, Bogotá, v. 15, n. 3, p. 479-496, 2012. Disponible en: <http://educacionyeducadores. unisabana.edu.co/index.php/eye/article/view/1962>. Acceso en: 24 my. 2018.

CARDONA, José. Hacia la mejora de la formación práctica del estudiante de pedagogía en la UNED. Educación XXI, Madrid, n. 14, n. 2, p. 303-330, 2011.

CASTELLANOS, Sonia; YAYA, Ruby. La reflexión docente y la construcción de conocimiento: una experiencia desde la práctica. Sinéctica, Jalisco, n. 41, p. 2-18, 2013. Disponible en: <http://www.scielo.org.mx/ scielo.php?script=sci_arttext\&pid=S1665-109X2013000200006 >. Acceso en: 24 my. 2018.

COFFEY, Amanda; ATKINSON, Paul. Encontrar el sentido a los datos cualitativos: estrategias complementarias de investigación. Medellín: Universidad de Antioquia, 2003.

CORNEJO A. José; FUENTEALBA J. Rodrigo. Prácticas reflexivas para la formación profesional docente: ¿qué las hace eficaces? Santiago de Chile: Universidad Católica Silva Henríquez, 2008.

DIAZ, Víctor. Formación docente, práctica pedagógica y saber pedagógico. Laurus, Caracas, v. 12, n. extra, p. 88-103, 2006.

DOMINGO ROGET, Àngels; GÓMEZ SERÉS, M. Victoria. La práctica reflexiva: bases, modelos e instrumentos. Madrid: Narcea, 2014.

ERAZO, María. Práctica reflexiva como estrategia de desarrollo profesional: presencia y estructura en reuniones docentes. Revista Educación y Educadores, Bogotá, v. 12, n. 2, p. 47-74, 2009. Disponible en: $<$ http://educacionyeducadores.unisabana.edu.co/index.php/eye/article/view/1485/1654>. Acceso en: 24 my. 2018.

ETSCHEIDT, Susan; CURRAN, Christina; SAWYER, Candace. Promoting Reflection in teacher preparation programs: a multilevel model. Teacher Education and Special Education, Florida, v. 35, n. 1, p. 7-26, 2012. Disponible en: <http://journals.sagepub.com/doi/abs/10.1177/0888406411420887>. Acceso en: 24 my. 2018.

FLORES, Rafael. Pedagogía del conocimiento. Bogotá: McGraw-Hill, 2005.

FLORES, Rodrigo. Observando observadores: una introducción a las técnicas cualitativas de investigación social. Santiago de Chile: Universidad Católica de Chile, 2009. 
FRAILE, Antonio. El profesorado de educación física en la sociedad del conocimiento. Tándem: Didáctica de la Educación Física, Barcelona, v. 36, p. 45-52, 2011.

FRANCIS, Susan; MARÍN, Patricia. Hacia la construcción del saber pedagógico en las comunidades académicas: un estudio desde la opinión de docentes universitarios. Actualidades Investigativas en Educación, San José, v. 10, n. 2, p. 1-29, 2010. Disponible en: <https://revistas.ucr.ac.cr/index.php/aie/ article/view/10134/9535>. Acceso en: 24 my. 2018.

FREIRE, Leila; FERNANDEZ, Carmen. 0 professor universitário novato: tensões, dilemas e aprendizados no início da carreira docente. Ciência \& Educação, Bauru, v. 21, n. 1, p. 255-272, 2015. Disponible en: <http:// www.scielo.br/pdf/ciedu/v21n1/1516-7313-ciedu-21-01-0255.pdf>. Acceso en: 24 my. 2018.

FREIRE, Paulo. Pedagogía de la esperanza, un reencuentro con la pedagogía del oprimido. Buenos Aires: Siglo XXI, 2002.

FULLAN, Michael; HARGREAVES, Andy. La escuela que queremos: los objetivos por los cuales vale la pena luchar. Buenos Aires: Editorial Madrid, 2006.

GARRID0, Carmen. El problema de la reflexión en la universidad a partir del relato de una experiencia en educación. Revista Internacional de Aprendizaje en la Educación Superior, Madrid, v. 3, n. 2, p. 167-175, 2016. Disponible en: <http://journals.epistemopolis.org/index.php/edusuperior/article/ download/255/925>. Acceso en: 24 my. 2018.

GIBBS, Graham. El análisis de datos cualitativos en investigación cualitativa. Madrid: Morata, 2012.

GRAHAM, Daniel; GREG, Auhl; HASTINGS, Wendy. Collaborative feedback and reflection for professional growth: preparing first- year pre-service teachers for participation in the community of practice. AsiaPacific Journal of Teacher Education, Brisbane, v. 41, n. 2, p. 159-172, 2013. Disponible en: <https://www. tandfonline.com/doi/abs/10.1080/1359866X.2013.777025>. Acceso en: 24 May. 2018.

INFANTE, Marta. Desafíos a la formación docente: inclusión educativa. Estudios Pedagógicos, Valdivia, v. 36, n. 1, p. 287-297, 2010. Disponible en: <http://mingaonline.uach.cl/pdf/estped/v36n1/art16.pdf>. Acceso en: 24 my. 2018.

KVALE, Steinar. Las entrevistas en investigación cualitativa. Madrid: Morata, 2011.

LATORRE, Marisol. Saber pedagógico en uso: características del saber actuante en las prácticas pedagógicas. Santiago de Chile: PUCC, 2002. 235 p. Tesis (Doctorado en Ciencias de la Educación) - Facultad de Educación de la Pontificia Universidad Católica de Chile y a l'Ecole Doctorale de la Faculté des Sciences Humaines et Sociales de I'Université Paris 5 - René Descartes, Santiago de Chile [y] Sorbonne, 2002.

MARCELO, Carlos. Desenvolvimiento profissional docente: passsado e futuro. SISIF0, Lisboa, n. 8, p. 7-22, jan./abr. 2009. Disponible en: <https://idus.us.es/xmlui/handle/11441/29247>. Acceso en: 24 my. 2018.

MATURANA, Humberto. Transformación en la convivencia. Santiago de Chile: J. C. Saez, 2011. 
MAURI, Teresa; CLARÁ, Marc; ONRUBIA, Javier. Intervención educativa para mejorar la práctica reflexiva en estudiantes de Magisterio. Electronic Journal of Research in Educational Psychology, Almería, v. 14, n. 2, p. 287-309, 2016. Disponible en: <http://ojs.ual.es/ojs/index.php/EJREP/article/view/1686>. Acceso en: 24 my. 2018.

MENA, Juan; GARCÍA, María. El papel de la reflexión crítica en la construcción de conocimiento práctico docente. Tendencias Pedagógicas, Madrid, v. 22, p. 197-210, 2013.

MONTENEGRO, Helena; FUENTEALBA, Adrián. El formador de futuros profesionales: una nueva forma de comprender la docencia en la educación superior universitaria. Calidad en la Educación, Santiago de Chile, n. 32, p. 253-267, 2010.

MONTENEGRO, Vanda; FERNANDEZ, Carmen. Processo reflexivo e desenvolvimento do conhecimento pedagógico do conteúdo numa intervenção formativa com professores de química. Ensaio, Belo Horizonte, v. 17, n. 1, p. 251-275, 2015. Disponible en: <https://dialnet.unirioja.es/servlet/articulo?codigo=3731344>. Acceso en: 24 my. 2018.

MOORE-RUSSO, Deborah; WILSEY, Jilian. Delving into the meaning of productive reflection: a study of future teacher's reflections on representations of teaching. Teaching and Teacher Education, Oxford, v. 37, p. 7690, 2014. Disponible en: <https://www.sciencedirect.com/science/article/pii/S0742051X13001509>. Acceso en: 24 my. 2018.

MUÑOZ, María; ALMONACID, Alejandro. Cognición, juego y aprendizaje: una propuesta para el aula de la primera infancia. Infancia, Educación y Aprendizaje, Valparaíso, v. 1 n. 1, p. 162-177. Disponible en: $<$ http://revistas.uv.cl/index.php/IEYAarticle/view/576/592>. Acceso en: 18 jun. 2018.

RODRÍGUEZ, Gregorio; GIL, Javier; GARCÍA, Eduardo. Metodología de la investigación cualitativa. Málaga: Aljibe, 1999.

RUFFINELLI, Andrea. Formación de docentes reflexivos: un enfoque en construcción y disputa. Educação e Pesquisa, São Paulo, v. 43, n. 1, p. 97-111, 2017. Disponible en: <http://www.scielo.br/pdf/ep/ v43n1/1517-9702-ep-43-1-0097.pdf>. Acceso en: 24 my. 2018.

SANTANA, Rosane; DALMAZO, Marli. 0 professor formador e as relações com os seus saberes profissionais. Revista Diálogo Educativo, Santiago de Chile, v. 12, n. 36, p. 511-527, 2012.

SANTIAGO, María; PARRA José; MURRILLO, Misael. Docente intelectual: gestor de la reflexión crítica. Perfiles Educativos, Ciudad de México, v. 34, n. 137, p. 164-178, 2012. Disponible en: <http://www2. pucpr.br/reol/index.php/dialogo?dd1=6098\&dd2=4745\&dd3=\&dd99=pdf>. Acceso en: 24 my. 2018.

SANTORO, María. Entre a lógica de formação e a lógica das práticas: a mediação dos saberes pedagógicos. Educação e Pesquisa, São Paulo, v. 34, n. 1, p. 109-126, 2008. Disponible en: <http://www.revistas.usp. br/ep/article/view/28079 >. Acesso en: 24 my. 2018.

SCHÖN, Donald. El profesional reflexivo: cómo piensan los profesionales cuando actúan. Barcelona: Paidós, 1998. 
SCHÖN, Donald. La formación de profesionales reflexivos: hacia un nuevo diseño de la enseñanza y el aprendizaje en las profesiones. Madrid: Paidós, 2010.

SIMONS, Helen. El estudio de caso: teoría y práctica. Madrid: Morato, 2011.

STRAUSS, Anselm; CORBIN, Juliet. Bases de la investigación cualitativa: técnicas y procedimientos para desarrollar la teoría fundamentada. Medellín: Universidad de Antioquia, 2002.

TARDIF, Maurice. Los saberes del docente y su desarrollo profesional. Madrid: Narcea, 2004.

TAYLOR, Steven J; BOGDAN Robert. Introducción a los métodos cualitativos de investigación. Barcelona: Paidós, 1984.

VASILACHIS, Irene Vasilachis de. Estrategias de investigación cualitativa. Barcelona: Gedisa, 2006.

Recibido en: 26.02.2018 Revisiones en: 16.05 .2018 Aprobado en: 13.06.2018

Eugenio Merellano-Navarro es doctor en Actividades Artísticas y Físicas, docente investigador de la Facultad de Educación, Universidad Autónoma de Chile. Experiencia como investigador en el área de la Didáctica de la Educación Física.

Alejandro Almonacid-Fierro es doctor en Actividades Artísticas y Físicas, Docente Investigador, Facultad de Educación, Universidad Católica del Maule, Chile. Experiencia como investigador en el área de formación del profesorado.

María Francisca Muñoz Oyarce es magíster en Educación Diferencial y Psicopedagogía, Facultad de Educación, Universidad Autónoma de Chile. 